\title{
Nasal Swab or Nose Specimen
}

National Cancer Institute

\section{Source}

National Cancer Institute. Nasal Swab or Nose Specimen. NCI Thesaurus. Code C155833.

A biospecimen collected from the nasal passages by swabbing. 